\title{
Repurposing and Revival of the Drugs: A New Approach to Combat the Drug Resistant Tuberculosis
}

\author{
Divakar Sharma ${ }^{1,2 *}$, Yogesh K. Dhuriya ${ }^{3}$, Nirmala Deo ${ }^{1}$ and Deepa Bisht ${ }^{1}$ \\ 'Department of Biochemistry, National JALMA Institute for Leprosy and Other Mycobacterial Diseases, Agra, India, \\ ${ }^{2}$ Interdisciplinary Biotechnology Unit, Aligarh Muslim University, Aligarh, India, ${ }^{3}$ Developmental Toxicology Laboratory, \\ Systems Toxicology and Health Risk Assessment Group, CSIR-Indian Institute of Toxicology Research (CSIR-IITR), Lucknow, \\ India
}

Emergence of drug resistant tuberculosis like multi drug resistant tuberculosis (MDR-TB), extensively drug-resistant tuberculosis (XDR-TB) and totally drug resistant tuberculosis (TDR-TB) has created a new challenge to fight against these bad bugs of Mycobacterium tuberculosis. Repurposing and revival of the drugs are the new trends/options to combat these worsen situations of tuberculosis in the antibiotics resistance era or in the situation of global emergency. Bactericidal and synergistic effect

OPEN ACCESS

Edited by:

Noton Kumar Dutta,

Johns Hopkins University,

United States

Reviewed by:

Sandeep Sharma,

University of Pennsylvania,

United States

Shashank Gupta,

Brown University, United States

*Correspondence:

Divakar Sharma

divakarsharma88@gmail.com

Specialty section:

This article was submitted to

Antimicrobials, Resistance

and Chemotherapy,

a section of the journal

Frontiers in Microbiology

Received: 29 September 2017 Accepted: 27 November 2017

Published: 11 December 2017

Citation:

Sharma D, Dhuriya YK, Deo $N$ and

Bisht D (2017) Repurposing and Revival of the Drugs: A New Approach to Combat the Drug

Resistant Tuberculosis.

Front. Microbiol. 8:2452.

doi: 10.3389/fmicb.2017.02452 of repurposed/revived drugs along with the latest drugs bedaquiline and delamanid used in the treatment of MDR-TB, XDR-TB, and TDR-TB might be the choice for future promising combinatorial chemotherapy against these bad bugs.

Keywords: drug resistance tuberculosis, repurposing, revival of drugs, synergistic effect, proteomics and bioinformatics

\section{INTRODUCTION}

\section{Current Scenario}

Mycobacterium tuberculosis is a deadly infectious pathogen causing tuberculosis (TB) worldwide. According to WHO (2016) 10.4 million new cases with 1.5 million deaths including 0.4 million individuals with HIV-TB co-infection were reported globally (WHO, 2016). Although TB can be cured by chemotherapy, but the emergence of drug resistant tuberculosis [such as multidrug-resistant tuberculosis (MDR-TB), extensively drug-resistant tuberculosis (XDR$\mathrm{TB}$ ) and totally drug resistant tuberculosis (TDR-TB)] has created a new challenge to combat the adverse situation of the disease. Since the decade rates of antibiotic resistance to first and second line anti-TB drugs are dramatically increasing (Cambau et al., 2015). Due to various complexities and high burden of HIV-TB co-infection treatments of MDR-TB, XDR$\mathrm{TB}$, and TDR-TB are problematic. Apart from the genomics studies various proteomics as well as bioinformatics studies regarding to the drug resistance tuberculosis were accumulated in the last decade (Jiang et al., 2007; Zhang and Yew, 2009; Sharma et al., 2010, 2014, 2015a,b, 2016a,b, 2017; Kumar et al., 2013; Lata et al., 2015a,b; Singh et al., 2015; Sharma and Bisht, 2016, 2017a,b,c;) and suggested the novel drug resistance mechanisms/markers/targets for potential therapeutics in near future. Proteomics and bioinformatics play a crucial role in the diagnostics and therapeutics against the emerging bad bug of tuberculosis. Apart from the expression proteomics studies cited above we have reported that Rv0148 (Putative shortchain type dehydrogenase/reductase) and Rv3841 (ferritin protein) have potentially involved in aminoglycosides resistance (Sharma et al., 2015b, 2016a) and could be the potential diagnostics and 
therapeutics against the second line of drug resistance. To combat these worsened situations (MDR-TB, XDR-TB, and TDR-TB) new and effective drugs and diagnostics are urgently needed. However, as we know that development of new drugs and diagnostics are enormously expensive and time consuming process.

\section{Repurposing and Revival of Drugs: A Chemotherapeutic Option against Drug Resistant Tuberculosis}

Repurposing and revival of the drugs are the new trends/options or one of the pharmaceutical strategies to treat the particular disease that are already FDA approved for other diseases (Tsukamura, 1980; Van Deun et al., 2010) and also for tuberculosis earlier, in this antibiotics resistance era or in the situation of global emergency. Many compounds in TB advanced clinical trials are the molecules that were formerly used to treat other infectious diseases/tuberculosis earlier and now they have been repurposed for treatment of TB (Nayer and Steinbach, 1939; Tsukamura, 1980; Van Deun et al., 2010; Hasse et al., 2014).

Sulfonamides and sulphanilamide were first used in 1930s as an anti-TB drug (Nayer and Steinbach, 1939) but its use was discontinued due to its lesser efficacy as compared to first line drugs (streptomycin and isoniazid). Revival of sulfamethoxazole (SMX) in TB was first pointed out by its efficacy to prevent the TB infection in HIV patients whose receiving trimethoprim/sulfamethoxazole (TMP/SMX) to prevent other infection such as Pneumocystis jirovecii (Hasse et al., 2014). In a Nigerian trial study on patients of HIV-MDR-TB coinfetion, efficiency of MDR-TB treatment by TMP/SMX confirmed a significantly shorter time to sputum conversion in these patients (Oladimeji et al., 2014). Sulfadiazine is an anti-leprosy drug which is repurposed in the treatment of MDR-TB and XDR-TB. Brouqui et al. (2013) suggested that sulfadiazine regimen is safe and effective against MDR-TB and TDR-TB treatment (Ameen and Drancourt, 2013; Brouqui et al., 2013).

Clofazimine (CZM) is one of the repurposed molecules, has been initially used as an anti-leprosy drug since half the century. It was recently repurposed for managing the treatment of MDRTB (Van Deun et al., 2010). CZM is recommended as a secondline anti-TB drug and used in combination with other anti-TB drugs for the treatment of drug-resistant tuberculosis. CZMcontaining regimen can cure MDR-TB cases in 9-12 months. In M. tuberculosis, CZM appears to act as a prodrug, which is reduced by type 2-NADH dehydrogenase, to release reactive oxygen species (ROS) upon reoxidation by oxygen $\left(\mathrm{O}_{2}\right)$ (Yano et al., 2011). CZM, exhibits noticeable anti-mycobacterial and anti-inflammatory activity by inhibition of phospholipase and effects on potassium transporters, respectively (Steele et al., 1999; Cholo et al., 2006). Previous published studies have reported that CZM has good quality efficacy and little toxicity against drug-resistant mycobacterial strains in animal models, which suggested, CZM as a promising anti-TB drug for the management of MDR-TB (Van Deun et al., 2010; Cholo et al., 2012). Recently, numerous observational studies have reported that CZM including regimens provided a useful role in the treatment of patients with MDR-TB (Cholo et al., 2006; Van Deun et al., 2010).

Linezolid, an oxazolidinone antibiotic which is used for treatment of gram-positive bacterial infections (Till et al., 2002; Yanagihara et al., 2002), has now potentially repurposed for the treatment of drug resistant TB (MDR-TB and XDR-TB) (Fortún et al., 2005). Linezolid is an effective anti-TB drug for treating MDR-TB and XDR-TB with various side effects such as neurotoxicity and hematologic toxicity (Tang et al., 2015). Schecter et al. (2010) suggested that linezolid had low rates of discontinuation, well tolerated and good efficacy in the treatment of MDR-TB. Most recently in a case study Jaspard et al. (2017) reported, bedaquiline and linezolid drug combination might be safe for XDR-TB in the late third trimester of pregnancy or pregnant woman. Pregnant woman gave birth to a child without abnormalities follow-up of the fetal showed that no fetal toxicities upto 2 years after the delivery (Jaspard et al., 2017).

Minocycline is also one of the repurposed molecules, has been initially used in the treatment of leprosy since the 1980s (Tsukamura, 1980). In 2008 it was repurposed for managing the treatment of XDR-TB patient in Japan (Kawada et al., 2008). Combinatorial therapy of amoxicillin/clavulanic acid along with other second-line drugs has been used in the treatment of MDR-TB. It's cheaper cost and less toxicity has made the drug of choice in WHO group five drugs (Cassir et al., 2014). Recently, combinatorial treatment by amoxicillin/clavulanic acid and carbapenems has reduced the M. tuberculosis load (Diacon et al., 2016). Hugonnet et al. (2009) reported the in vitro activity of meropenem combined with clavulanate against XDR strains and paying attention to repurpose these beta-lactams as new anti-TB drugs (Hugonnet et al., 2009). However, carbapenems have been used successfully as part of salvage therapies for XDR patients, they have to be administered intravenously (Tiberi et al., 2016). Recently, an early bactericidal activity-Phase II (EBA Phase II) clinical trial has validated the promising potential of a carbapenem combined with amoxicillin and clavulanic acid for TB treatment (Diacon et al., 2016).

Singhal et al. (2014) reported that the FDA-approved antidiabetic drug metformin (MET) inhibits the intracellular mycobacterial growth by inducing mitochondrial ROS production, restricts disease immunopathology, enhances the efficacy of other anti-TB drugs and could be used as combinatorial therapy against the drug resistant tuberculosis. Gupta et al. (2013) suggested that tuberculosis treatment shortening by verapamil as an adjunctive therapy in mice has opened the direction for future research on verapamil and other efflux pump inhibitors in human tuberculosis. Gupta et al. (2014) also reported that efflux inhibition with verapamil tremendously decreases the MIC of bedaquiline and CZM to M. tuberculosis and suggested the synergistic effects of verapamil and bedaquiline in an animal model of TB infection. Recently Dutta et al. (2016) reported that statin a lipid-lowering drug (repurposed for TB treatment) when added to the first-line antitubercular drugs, reduces the lung bacillary burden in chronically infected mice. 
Host-directed therapies are important adjunctive therapies for tuberculosis treatment that expanded by host immune effector mechanisms. Recently Gupta et al. (2017) showed that denileukin diftitox potentiates standard TB treatment in the mouse model, which might be due to depletion of T-regulatory and myeloidderived suppressor cells during TB infection.

\section{Synergistic Effects of Repurposed/Revived Drugs: With Others Anti-TB Drugs Used in Treatments of Tuberculosis Bad Bug}

Synergistic effect of repurposed/revived drugs with other antiTB drugs has been used for the future selection of these drugs in the WHO regimen which could be used in the treatment of MDR-TB, XDR-TB, and TDR-TB. In a study Zhang et al. (2015) suggested that CZM in combination with ethambutol (EMB) and moxifloxacin (MOX) may be a potential drug regimen for the treatment of MDR-TB. Synergistic effect of SMX has been reported in vitro with rifampicin (Macingwana et al., 2012). Tasneen et al. (2011) suggested CZM was the best third drug in combination with bedaquiline and pyrazinamide for the treatment of MDR-TB and a good example of drug synergism. Partial synergistic effect was oveserved between linezolid and capreomycin and suggested the efficacy of this combinatorial therapy against M. tuberculosis (Zhao et al., 2016). Most recently synergistic effect of bedaquiline and linezolid combinatorial therapy has been reported in XDR-TB of pregnant woman which is a good symbol of synergy for the last line of drugs (Jaspard et al., 2017). In a study synergistic effect carbapenems with rifampicin have been reported against $M$. tuberculosis (Kaushik et al., 2015).

\section{REFERENCES}

Ameen, S. M., and Drancourt, M. (2013). In vitro susceptibility of Mycobacterium tuberculosis to trimethoprim and sulfonamides in France. Antimicrob. Agents Chemother. 57, 6370-6371. doi: 10.1128/AAC.01 683-13

Brouqui, P., Aubry, C., Million, M., Drancourt, M., and Raoult, D. (2013). Totally resistant tuberculosis: Will antileprosy drugs be helpful? Int. J. Antimicrob. Agents 42, 584-585. doi: 10.1016/j.ijantimicag.2013. 08.004

Cambau, E., Viveiros, M., Machado, D., Raskine, L., Ritter, C., Tortoli, E., et al. (2015). Revisiting susceptibility testing in MDR-TB by a standardized quantitative phenotypic assessment in a European multicentre study. J. Antimicrob. Chemother. 70, 686-696. doi: 10.1093/jac/ dku 438

Cassir, N., Rolain, J. M., and Brouqui, P. (2014). A new strategy to fight antimicrobial resistance: the revival of old antibiotics. Front. Microbiol. 5:551. doi: $10.3389 /$ fmicb.2014.00551

Cholo, M., Boshoff, H. I., Steel, H. C., Cockeran, R., Matlola, N. M., Downing, K. J., et al. (2006). Effects of clofazimine on potassium uptake by a Trk-deletion mutant of Mycobacterium tuberculosis. J. Antimicrob. Chemother. 57, 79-84. doi: $10.1093 /$ jac/dki409

Cholo, M. C., Steel, H. C., Fourie, P. B., Germishuizen, W. A., and Anderson, R. (2012). Clofazimine: current status and future prospects. J. Antimicrob. Chemother. 67, 290-298. doi: 10.1093/jac/dkr444

Diacon, A. H., van der Merwe, L., Barnard, M., von Groote-Bidlingmaier, F., Lange, C., García-Basteiro, A. L., et al. (2016). beta-Lactams against

\section{CONCLUSION AND FUTURE PERSPECTIVE}

On the basis of the reported literature and studies (regarding to proteomics, bioinformatics, and repurposing/revival of drugs) cited above we suggest that proteomics and bioinformatics play a crucial role in the exploration of diagnostics, therapeutics and mechanism of resistance against drug resistance tuberculosis. Repurposing and revival of the drugs is an alternative strategy to combat from this inadequate situation of drug resistance tuberculosis in this antibiotic resistance era. Synergistic effect of repurposed/revived drugs (sulfamethoxazole, sulfadiazine, clofazimine, linezolid, minocycline, amoxicillin/clavulanic acid, and carbapenems like meropenem) along with the latest drugs (bedaquiline and delamanid) used in the treatment of MDRTB and XDR-TB might be one of the promising combinatorial chemotherapy for the treatment of MDR-TB, XDR-TB, and TDR-TB. Synergistic effect of these repurposed/revived drugs with bedaquiline and delamanid combinatorial therapy could be added a different perspective over the existed literature.

\section{AUTHOR CONTRIBUTIONS}

DS design the concept and wrote the manuscript. DS, YD, ND, and DB finalized the manuscript.

\section{ACKNOWLEDGMENTS}

The authors are grateful to Director, NJIL \& OMD for the support. DS is ICMR-PDFs (ICMR, New Delhi).

Tuberculosis-new trick for an old dog? N. Engl. J. Med. 375, 393-394. doi: 10.1056/NEJMc1513236

Dutta, N. K., Bruiners, N., Pinn, M. L., Zimmerman, M. D., Prideaux, B., Dartois, V., et al. (2016). Statin adjunctive therapy shortens the duration of TB treatment in mice. J. Antimicrob. Chemother. 71, 1570-1577. doi: 10.1093/jac/ dkw014

Fortún, J., Martín-Dávila, P., Navas, E., Pérez-Elías, M. J., Cobo, J., Tato, M., et al. (2005). Linezolid for the treatment of multidrug-resistant tuberculosis. J. Antimicrob. Chemother. 56, 180-185. doi: 10.1093/jac/ dki148

Gupta, S., Cheung, L., Pokkali, S., Winglee, K., Guo, H., Murphy, J. R., et al. (2017). Suppressor cell-depleting immunotherapy with denileukin diftitox is an effective host-directed therapy for tuberculosis. J. Infect. Dis. 215, 1883-1887. doi: 10.1093/infdis/jix208

Gupta, S., Cohen, K. A., Winglee, K., Maiga, M., Diarra, B., and Bishai, W. R. (2014). Efflux inhibition with verapamil potentiates bedaquiline in Mycobacterium tuberculosis. Antimicrob. Agents Chemother. 58, 574-576. doi: 10.1128/AAC.01462-13

Gupta, S., Tyagi, S., Almeida, D. V., Maiga, M. C., Ammerman, N. C., and Bishai, W. R. (2013). Acceleration of tuberculosis treatment by adjunctive therapy with verapamil as an efflux inhibitor. Am. J. Respir. Crit. Care Med. 188, 600-607. doi: 10.1164/rccm.201304-0650OC

Hasse, B., Walker, A. S., Fehr, J., Furrer, H., Hoffmann, M., Battegay, M., et al. (2014). Cotrimoxazole prophylaxis is associated with reduced risk of incident tuberculosis in participants in the Swiss HIV Cohort Study. Antimicrob. Agents Chemother. 58, 2363-2368. doi: 10.1128/AAC. 01868-13 
Hugonnet, J. E., Tremblay, L. W., Boshoff, H. I., Barry, C. E. III, and Blanchard, J. S. (2009). Meropenem-clavulanate is effective against extensively drugresistant Mycobacterium tuberculosis. Science 323, 1215-1218. doi: 10.1126/ science. 1167498

Jaspard, M., Elefant-Amoura, E., Melonio, I., De Montgolfier, I., Veziris, N., and Caumes, E. (2017). Bedaquiline and linezolid for extensively drug-resistant tuberculosis in pregnant woman. Emerg. Infect. Dis. 23, 1731-1732. doi: 10. 3201/eid2310.161398

Jiang, X., Zhang, W., Gao, F., Huang, Y., Lv, C., and Wang, H. (2007). Comparison of the proteome of isoniazid-resistant and -susceptible strains of Mycobacterium tuberculosis. Microb. Drug Resist. 12, 231-238. doi: 10.1089/ mdr.2006.12.231

Kaushik, A., Makkar, N., Pandey, P., Parrish, N., Singh, U., and Lamichhane, G. (2015). Carbapenems and rifampin exhibit synergy against Mycobacterium tuberculosis and Mycobacterium abscessus. Antimicrob. Agents Chemother. 59, 6561-6567. doi: 10.1128/AAC.01158-15

Kawada, H., Yamazato, M., Shinozawa, Y., Suzuki, K., Otani, S., Ouchi, M., et al. (2008). Achievement of sputum culture negative conversion by minocycline in a case with extensively drug-resistant pulmonary tuberculosis. Kekkaku 83, 725-728.

Kumar, B., Sharma, D., Sharma, P., Katoch, V. M., Venkatesan, K., and Bisht, D. (2013). Proteomic analysis of Mycobacterium tuberculosis isolates resistant to kanamycin and amikacin. J. Proteomics 94, 68-77. doi: 10.1016/j.jprot.2013.08.025

Lata, M., Sharma, D., Deo, N., Tiwari, P. K., Bisht, D., and Venkatesan, K. (2015a). Proteomic analysis of ofloxacin-mono resistant Mycobacterium tuberculosis isolates. J. Proteomics 127, 114-121. doi: 10.1016/j.jprot.2015. 07.031

Lata, M., Sharma, D., Kumar, B., Deo, N., Tiwari, P. K., Bisht, D., et al. (2015b). Proteome analysis of ofloxacin and moxifloxacin induced Mycobacterium tuberculosis isolates by proteomic approach. Protein Pept. Lett. 22, 362-371.

Macingwana, L., Baker, B., Ngwane, A. H., Harper, C., Cotton, M. F., Hesseling, A., et al. (2012). Sulfamethoxazole enhances the antimycobacterial activity of rifampicin. J. Antimicrob. Chemother. 67, 2908-2911. doi: 10.1093/jac/dks306

Nayer, H., and Steinbach, M. (1939). Sulfanilamide in clinical tuberculosis. Am. Rev. Tuberc. 40, 470-472.

Oladimeji, O., Isaakidis, P., Obasanya, O. J., Eltayeb, O., Khogali, M., Van den Bergh, R., et al. (2014). Intensive-phase treatment outcomes among hospitalized multidrug-resistant tuberculosis patients: results from a nationwide cohort in Nigeria. PLOS ONE 9:e94393. doi: 10.1371/journal.pone.00 94393

Schecter, G. F., Scott, C., True, L., Raftery, A., Flood, J., and Mase, S. (2010). Linezolid in the treatment of multidrug-resistant tuberculosis. Clin. Infect. Dis. 50, 49-55. doi: 10.1086/648675

Sharma, D., and Bisht, D. (2016). An efficient and rapid lipophilic proteins extraction from Mycobacterium tuberculosis $\mathrm{H} 37 \mathrm{Rv}$ for two dimensional gel electrophoresis. Electrophoresis 37, 1187-1190. doi: 10.1002/elps.2016 00025

Sharma, D., and Bisht, D. (2017a). Secretory proteome analysis of streptomycin resistant Mycobacterium tuberculosis clinical isolates. SLAS Discov. 22, 12291238. doi: $10.1177 / 2472555217698428$

Sharma, D., and Bisht, D. (2017b). M. tuberculosis hypothetical proteins and proteins of unknown function: Hope for exploring novel resistance mechanisms as well as future target of drug resistance. Front. Microbiol. 8:465. doi: 10.3389/ fmicb.2017.00465

Sharma, D., and Bisht, D. (2017c). Role of bacterioferritin \& ferritin in M. tuberculosis pathogenesis and drug resistance: a future perspective by interactomic approach. Front. Cell. Infect. Microbiol. 7:240. doi: 10.3389/fcimb. 2017.00240

Sharma, D., Deo, N., and Bisht, D. (2017). Proteomics and bioinformatics: a modern way to elucidate the resistome in Mycobacterium tuberculosis. J. Proteomics Bioinform. 10:e33. doi: 10.4172/jpb.10 $00 \mathrm{e} 33$

Sharma, D., Kumar, B., Lata, M., Joshi, B., Venkatesan, K., Shukla, S. et al. (2015a). Comparative proteomic analysis of aminoglycosides resistant and susceptible Mycobacterium tuberculosis clinical isolates for exploring potential drug targets. PLOS ONE 10:e0139414. doi: 10.1371/journal.pone.01 39414

Sharma, D., Lata, M., Faheem, M., Khan, A. U., Joshi, B., Venkatesan, K., et al. (2015b). Cloning, expression and correlation of Rv0148 to amikacin \& kanamycin resistance. Curr. Proteomics 12, 96-100. doi: 10.2174/ 157016461202150903113053

Sharma, D., Lata, M., Faheem, M., Khan, A. U., Joshi, B., Venkatesan, K., et al. (2016a). M. tuberculosis ferritin (Rv3841): potential involvement in Amikacin (AK) \& Kanamycin (KM) resistance. Biochem. Biophys. Res. Commun. 478, 908-912. doi: 10.1016/j.bbrc.2016.08.049

Sharma, D., Lata, M., Singh, R., Deo, N., Venkatesan, K., and Bisht, D. (2016b). Cytosolic proteome profiling of aminoglycosides resistant Mycobacterium tuberculosis clinical isolates using MALDI-TOF/MS. Front. Microbiol. 7:1816 doi: $10.3389 /$ fmicb. 2016.01816

Sharma, D., Shankar, H., Lata, M., Joshi, B., Venkatesan, K., and Bisht, D. (2014). Culture filtrate proteome analysis of aminoglycoside resistant clinical isolates of Mycobacterium tuberculosis. BMC Infect. Dis. 14(Suppl.):60. doi: 10.1186/14712334-14-S3-P60

Sharma, P., Kumar, B., Gupta, Y., Singhal, N., Katoch, V. M., Venkatesan, K., et al. (2010). Proteomic analysis of streptomycin resistant and sensitive clinical isolates of Mycobacterium tuberculosis. Proteome Sci. 8:59. doi: 10.1186/14775956-8-59

Singh, A., Gopinath, K., Sharma, P., Bisht, D., Sharma, P., Singh, N., et al. (2015). Comparative proteomic analysis of sequential isolates of Mycobacterium tuberculosis from a patient with pulmonary tuberculosis turning from drug sensitive to multidrug resistant. Indian J. Med. Res. 141, 27-45. doi: 10.4103/ 0971-5916.154492

Singhal, A., Jie, L., Kumar, P., Hong, G. S., Leow, M. K., Paleja, B., et al. (2014). Metformin as adjunct antituberculosis therapy. Sci. Transl. Med. 6:263ra159. doi: 10.1126/scitranslmed.3009885

Steele, H., Matlola, N. M., and Anderson, R. (1999). Inhibition of potassium transport and growth of mycobacteria exposed to clofazimine and B669 is associated with a calcium-independent increase in microbial phospholipase A2 activity. J. Antimicrob. Chemother. 44, 209-216. doi: 10.1093/jac/44. 2.209

Tang, S., Yao, L., Hao, X., Zhang, X., Liu, G., Liu, X., et al. (2015). Efficacy, safety and tolerability of linezolid for the treatment of XDR-TB: a study in China. Eur. Respir. J. 45, 161-170. doi: 10.1183/09031936.00 035114

Tasneen, R., Li, S., Peloquin, C., Taylor, D., Williams, K., Andries, K., et al. (2011). Sterilizing activity of novel TMC207- and PA-824-containing regimens in a murine model of tuberculosis. Antimicrob. Agents Chemother. 55, 5485-5492. doi: 10.1128/AAC.05293-11

Tiberi, S., D’Ambrosio, L., De Lorenzo, S., Viggiani, P., Centis, R., Sotgiu, G., et al. (2016). Ertapenem in the treatment of multidrug-resistant tuberculosis: first clinical experience. Eur. Respir. J. 47, 333-336. doi: 10.1183/13993003.012782015

Till, M., Wixson, R. L., and Pertel, P. E. (2002). Linezolid treatment for osteomyelitis due to vancomycin-resistant Enterococcus faecium. Clin. Infect. Dis. 34, 1412-1414. doi: 10.1086/340265

Tsukamura, M. (1980). In-vitro antimycobacterial activity of minocycline. Tubercle 61, 37-38. doi: 10.1016/0041-3879(80)90058-6

Van Deun, A., Maug, A. K., Salim, M. A., Das, P. K., Sarker, M. R., Daru, P., et al. (2010). Short, highly effective, and inexpensive standardized treatment of multidrug-resistant tuberculosis. Am. J. Respir. Crit. Care Med. 182, 684-692. doi: 10.1164/rccm.201001-0077OC

WHO (2016). Global Tuberculosis Control 2016. Available at: http://www.who.int/ tb/publications/global_report/en/

Yanagihara, K., Kaneko, Y., Sawai, T., Miyazaki, Y., Tsukamoto, K., Hirakata, Y., et al. (2002). Efficacy of linezolid against methicillin-resistant or vancomycininsensitive Staphylococcus aureus in a model of hematogenous pulmonary infection. Antimicrob. Agents Chemother. 46, 3288-3291. doi: 10.1128/AAC.46. 10.3288-3291.2002

Yano, T., Kassovska-Bratinova, S., The, J. S., Winkler, J., Sullivan, K., Isaacs, A., et al. (2011). Reduction of clofazimine by mycobacterial type $2 \mathrm{NADH}$ :quinone oxidoreductase: a pathway for the generation of bactericidal levels of reactive oxygen species. J. Biol. Chem. 286, 10276-10287. doi: 10.1074/jbc.M110.200501 
Zhang, Y., and Yew, W. W. (2009). Mechanisms of drug resistance in Mycobacterium tuberculosis. Int. J. Tuberc. Lung Dis. 13, 1320-1330.

Zhang, Z., Li, T., Qu, G., Pang, Y., and Zhao, Y. (2015). In vitro synergistic activity of clofazimine and other antituberculous drugs against multidrug-resistant Mycobacterium tuberculosis isolates. Int. J. Antimicrob. Agents 45, 71-75. doi: 10.1016/j.ijantimicag.2014.09.012

Zhao, W., Zheng, M., Wang, B., Mu, X., Li, P., Fu, L., et al. (2016). Interactions of linezolid and second-line anti-tuberculosis agents against multidrug-resistant Mycobacterium tuberculosis in vitro and in vivo. Int. J. Infect. Dis. 52, 23-28. doi: $10.1016 /$ j.ijid.2016.08.027
Conflict of Interest Statement: The authors declare that the research was conducted in the absence of any commercial or financial relationships that could be construed as a potential conflict of interest.

Copyright (C) 2017 Sharma, Dhuriya, Deo and Bisht. This is an open-access article distributed under the terms of the Creative Commons Attribution License (CC BY). The use, distribution or reproduction in other forums is permitted, provided the original author(s) or licensor are credited and that the original publication in this journal is cited, in accordance with accepted academic practice. No use, distribution or reproduction is permitted which does not comply with these terms. 